\title{
Interacting with Visual Interfaces on Mobile Devices
}

\author{
Invited Talk
}

\section{Luca Chittaro}

HCI Lab, Dept. of Math and Computer Science, University of Udine, Italy chittaro@dimi.uniud.it, http://hcilab.uniud.it

\begin{abstract}
This invited talk will discuss some major issues in developing visual interfaces for mobile devices as well as demonstrate a number of new mobile visual applications and interaction techniques that we have developed in domains as diverse as tourism, health \& fitness, navigation, emergency response, geospatial and architectural visualization. It will also deal with the issue of providing designers with new tools to study the behavior of users of these new mobile applications.
\end{abstract}

Keywords: human-computer interaction, mobile devices, visual interfaces, information visualization, user studies

\section{Contents of the talk}

Mobile devices such as mobile phones and PDAs are increasingly used to support information needs of users on the move. As a consequence, information spaces that have been traditionally available only to desktop and laptop users (e.g., documents, pictures, web pages, maps, large databases,...) are moving to small screens as well, presenting application designers with new challenges. Indeed, the technical limitations of mobile devices combined with the peculiar needs of users on-the-go require a careful design of applications that are specifically thought for mobile devices and users [1]. As an example, the common form factors of mobile devices constrain screen space to a small fraction of what is available on a desktop. A typical 240x320 pixels display of a PDA has less than 1/16 the area of a typical 1280x1024 desktop display (see Fig. 1). Such size limitation makes it extremely difficult for users to navigate information spaces that do not fit a single screen, unless appropriate techniques to simplify interaction and navigation are provided. 


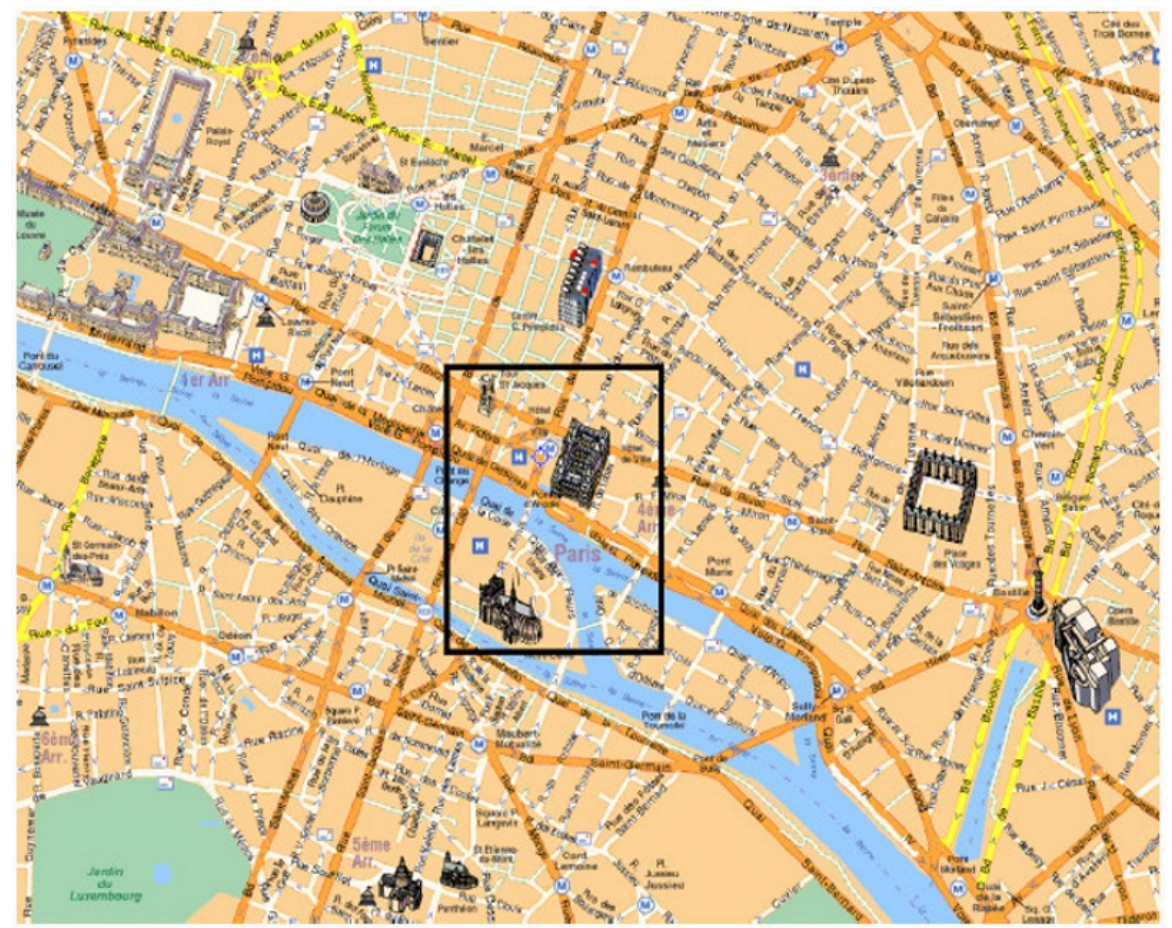

Figure 1 Comparison between the size of a typical 240x320 PDA screen (the area highlighted by the black rectangle) and a common 1280x1024 desktop screen (the whole picture).

The recent availability of mobile devices with increasingly powerful graphics capabilities is making it possible to develop novel visual interfaces, based on interactive 2D (or even 3D) graphics, to help users on the move in dealing more quickly and easily with larger amounts of information. Limited cognitive resources and safety of mobile users are an additional motivation to employ mobile graphics effectively as a way to provide information at-a-glance that is easily understood with less cognitive resources and distracts the user as less as possible from her surrounding environment.

Mobile visual interfaces become even more innovative and provide functionalities that were unavailable on desktop systems when they are integrated with various sensors (e.g., GPS, accelerometers, heart rate monitors, pulseoximeters,...) that allow one to adapt the behavior of the application according to position in space (location-awareness) and other parameters (context-awareness). In this way, the mobile device becomes able to choose what to show and how to show it on the display based on what is happening to the user as well as the physical world that surrounds her. For example, we developed a mobile personal trainer (see Fig. 2), 
called MOPET [2], that monitors user's position and physiological parameters in outdoor sports activities to present functionalities such as location-aware maps augmented with visualizations of users' performance or context-aware fitness advice and 3D demonstrations of exercises.

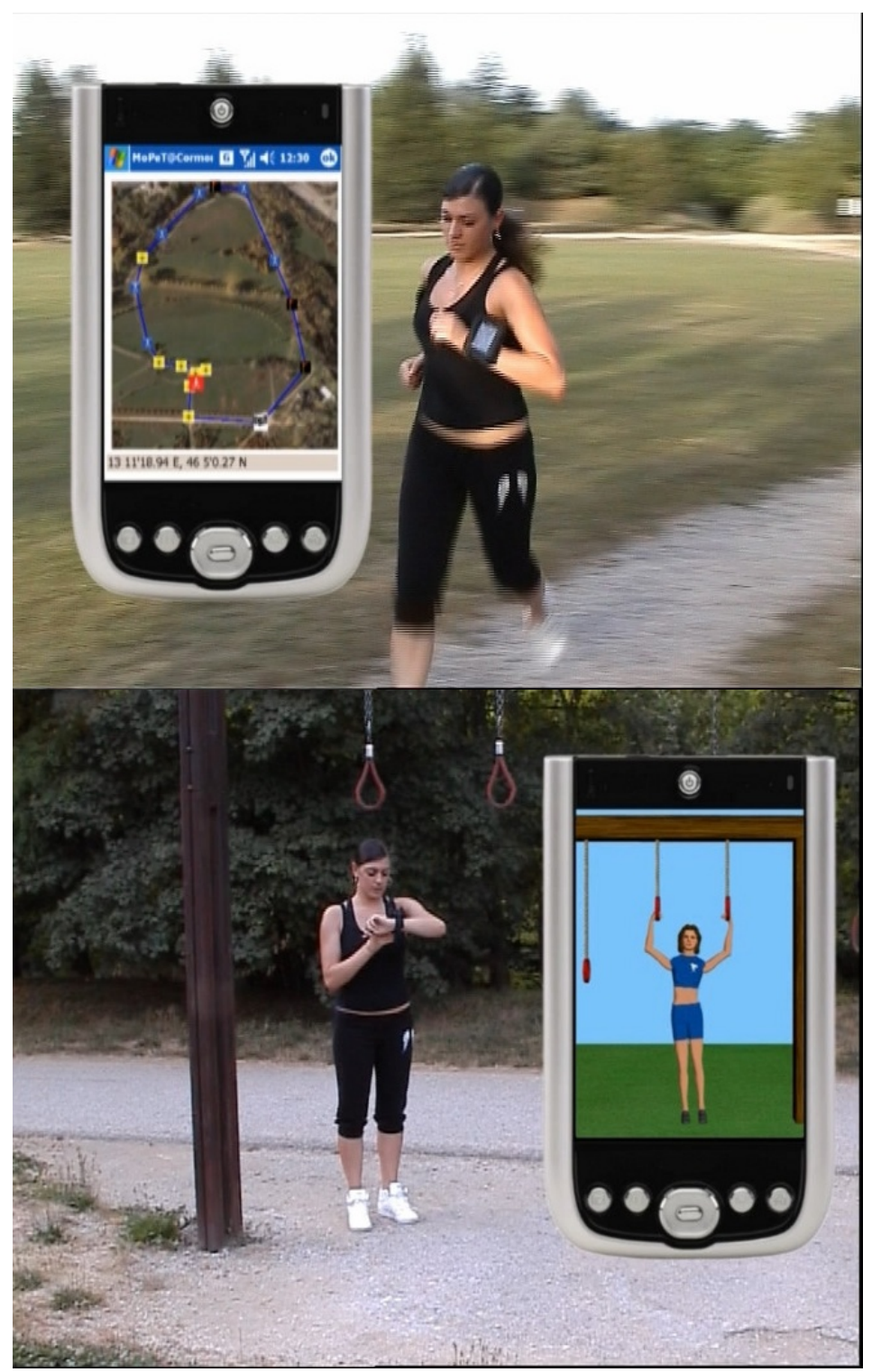

Figure 2 A wrist-worn context-aware system supports a user in outdoor fitness activities [2] 
This invited talk will discuss some major issues in developing visual interfaces for mobile devices as well as demonstrate a number of new mobile applications and interaction techniques that we have developed in domains as diverse as tourism[3], health \& fitness [2], navigation [4], emergency evacuation [5], geospatial and architectural visualization [6]. After an initial discussion of the peculiarities of the mobile context that motivate research, we will analyze some of the issues involved in designing a mobile visual interface, especially focusing on the so-called presentation problem $[1,7,8,9]$. The talk will illustrate a number of interaction and visualization solutions we have recently proposed and evaluated on users, such as the Zoom-Enhanced Navigator (ZEN) to explore information spaces on small screens [4], the MAGDA system to analyze datasets of geo-referenced elements in the field [3], and specific techniques for managing the off-screen objects [10] and the icon cluttering [11] problems. Then, location-awareness and contextawareness aspects will be introduced, considering very different application domains such as fitness training [2] or emergency response [5], and applications based on different kinds of sensors.

Finally, the talk will deal with the issue of providing designers with new tools to study the behavior of users of these new mobile applications. In particular, it will illustrate three different tools we have recently proposed for that purpose: the MOBREX tool [12] to log user interface actions on the mobile device and then visually analyze them on a desktop system, the VU-Flow [13] and the MOPET Analyzer [14] tools for the visual analysis of users' data collected through sensors connected to mobile devices.

\section{References}

1. Chittaro L. Visualizing Information on Mobile Devices, IEEE Computer, Vol. 39, No. 3, pp. 34-39 (2006).

2. Buttussi F., Chittaro L., MOPET: A Context-Aware and User-Adaptive Wearable System for Fitness Training. Artificial Intelligence In Medicine Journal, Vol. 42, No. 2, pp. 153163 (2008).

3. Burigat S., Chittaro L., Interactive Visual Analysis of Geographic Data on Mobile Devices based on Dynamic Queries, Journal of Visual Languages and Computing, Vol. 19, No. 1, pp. 99-122 (2008).

4. Burigat S., Chittaro L., Gabrielli S., Navigation Techniques for Small-screen Devices: an Evaluation on Maps and Web pages, International Journal of Human-Computer Studies, Vol. 66, No. 2, pp. 78-97 (2008).

5. Chittaro L., Nadalutti. D. Presenting Evacuation Instructions on Mobile Devices by means of Location-Aware 3D Virtual Environments, Proceedings of MOBILE HCI 2008: 10th International Conference on Human-Computer Interaction with Mobile Devices and Services, ACM Press, New York, in press (2008).

6. Mulloni A., Nadalutti D., Chittaro L., Interactive Walkthrough of Large 3D Models of Buildings on Mobile Devices, Proceedings of Web3D 2007: 12th International Conference on 3D Web Technology, ACM Press, New York, pp. 17-25 (2007). 
7. Gustafson, S., Baudisch, P., Gutwin, C, and Irani, P. Wedge: Clutter-Free Visualization of Off-Screen Locations, Proceedings of the CHI 2008 Conference on Human factors in computing systems, ACM Press, New York, pp. 787-796 (2008).

8. Bederson B.B., Clamage A., Czerwinski M.P., Robertson G.G. DateLens: A fisheye calendar interface for PDAs, ACM Transactions on Computer-Human Interaction, vol. 11, no.1, pp. 90-119 (2004).

9. Ware C. Information Visualization: Perception for Design, 2nd Edition, Morgan Kaufmann, San Mateo, CA, (2004).

10. Burigat S., Chittaro L., Gabrielli S., Visualizing Locations of Off-Screen Objects on Mobile Devices: A Comparative Evaluation of Three Approaches, Proceedings of MOBILE HCI 2006: 8th International Conference on Human-Computer Interaction with Mobile Devices and Services, ACM Press, New York, pp. 239-246 (2006).

11. Burigat S., Chittaro L., Decluttering of Icons based on Aggregation in Mobile Maps, In Meng L., Zipf A., Winter S. (eds), Map-based Mobile Services - Design, Interaction and Usability, Springer, Berlin, pp. 13-32 (2008).

12. Burigat S., Chittaro L., Ieronutti L., Mobrex: Visualizing Users' Mobile Browsing Behaviors, IEEE Computer Graphics and Applications, Vol. 28, No. 1, pp. 24-32 (2008).

13. Chittaro L., Ranon R., Ieronutti L., VU-Flow: A Visualization Tool for Analyzing Navigation in Virtual Environments, IEEE Transactions on Visualization and Computer Graphics, Special Issue on Visual Analytics, Vol. 12, No. 6, pp. 1475-1485 (2006).

14. Nadalutti, D., Chittaro, L. Visual Analysis of Users' Performance Data in Fitness Activities, Computers \& Graphics, Special Issue on Visual Analytics, Vol. 31, No. 3, pp. 429-439 (2007). 